\title{
Site-to-Site Internet Traffic Control
}

\author{
Frank Cangialosi*, Akshay Narayan* Prateesh Goyal , \\ Radhika Mittal $\$$, Mohammad Alizadeh , Hari Balakrishnan \\ MIT CSAIL * UIUC
}

\begin{abstract}
Queues allow network operators to control traffic: where queues build, they can enforce scheduling and shaping policies. In the Internet today, however, there is a mismatch between where queues build and where control is most effectively enforced; queues build at bottleneck links that are often not under the control of the data sender. To resolve this mismatch, we propose a new kind of middlebox, called Bundler. Bundler uses a novel inner control loop between a sendbox (in the sender's site) and a receivebox (in the receiver's site) to determine the aggregate rate for the bundle, leaving the endto-end connections and their control loops intact. Enforcing this sending rate ensures that bottleneck queues that would have built up from the bundle's packets now shift from the bottleneck to the sendbox. This enables the sendbox to exercise control over its traffic by scheduling packets according to any policy necessary to achieve the network operator's higherlevel objectives. We have implemented Bundler in Linux and evaluated it with real-world and emulation experiments. We find that Bundler allows the sender-chosen policy to be effective: when configured to implement Stochastic Fairness Queueing (SFQ), it improves median flow completion time (FCT) by between $28 \%$ and $97 \%$ across various scenarios.
\end{abstract}

\section{INTRODUCTION}

This paper introduces the idea of site-to-site Internet traffic control. By "site", we mean a single physical location with tens to many thousands of endpoints sharing access links to the rest of the Internet. Examples of sites include a company office, a coworking office building, a university campus, a single datacenter, and a point-of-presence $(\mathrm{PoP})$ of a regional Internet Service Provider (ISP).

Consider a company site with employees running thousands of concurrent applications. The administrator may wish to enforce certain traffic control policies for the company; for

${ }^{*}$ Both authors contributed equally to this work.

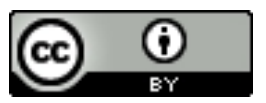

This work is licensed under a Creative Commons Attribution International 4.0 License EuroSys '21, April 26-28, 2021, Online, United Kingdom

(C) 2021 Copyright held by the owner/author(s). ACM ISBN 978-1-4503-8334-9/21/04.

https://doi.org/10.1145/3447786.3456260 example, ensuring rates and priorities for Zoom sessions, deprioritizing bulk backup traffic, prioritizing interactive web sessions, and so on. There are two issues that stand in the way: first, the bottleneck for these traffic flows may not be in the company's network, and second, the applications could all be transiting different bottlenecks. So what is the company to do?

Cloud computing has made the second issue manageable. Because the cloud has become the prevalent method to deploy applications today, applications from different vendors often run from a small number of cloud sites (e.g., Amazon, Azure, etc.). This means that the network path used by these multiple applications serving the company's users are likely to share a common bottleneck; for example, all the applications running from Amazon's US-West datacenter, all the video sessions from a given Zoom datacenter, and so on. In this setting, by treating the traffic between the datacenter site and the company site as a single aggregate, the company's network administrator may be able to achieve their traffic control objectives.

But what about the first issue? The bottleneck for all the traffic between Amazon US-West and the company may not be the site's access link or at Amazon, but elsewhere, e.g., within the company's ISP; indeed, that may be the common case [12, $15,30,42]$. Unfortunately, the company cannot control traffic when the queues build inside its ISP. And the ISP can't help because it does not know what the company's objectives are. ${ }^{1}$

We propose a system, Bundler, that solves this problem. Bundler enables flexible control of a traffic bundle between a source site and a destination site by shifting the queues that would otherwise have accumulated elsewhere to the source's site (Figure 2) . It then schedules packets from this shifted queue using standard techniques $[11,14,17,18,35,38,39,44$, $46,48,52]$ to reduce mean flow-completion times, ensure low packet delays, isolate classes of traffic from each other, etc.

The key idea in Bundler is a control loop between the source and destination sites to calculate the dynamic rate for the bundle. Rather than terminate end-to-end connections at the sites, we leave them intact and develop an "inner loop" control method between the two sites that computes this rate. The inner control loop uses a delay-based congestion control algorithm that ensures high throughput, but controls self-inflicted queueing delays at the actual bottleneck. By avoiding queues at the bottleneck, the source site can prioritize latency-sensitive applications and allocate rates according to its objectives.

\footnotetext{
${ }^{1}$ Interdomain QoS mechanisms [7, 51] have not succeeded in the Internet despite years of effort.
} 


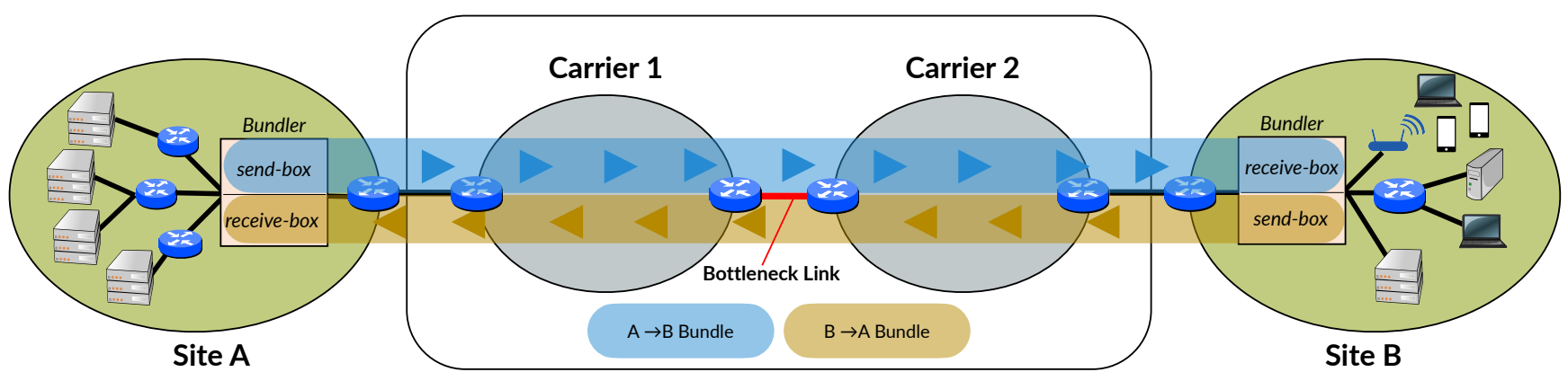

(e.g., AWS US-West datacenter)

Public Internet

(e.g., a company office network)

Figure 1: An example deployment scenario for Bundler in sites $A$ and $B$. Traffic between the two boxes is aggregated into a single bundle, shown as shaded boxes. The sendbox schedules the traffic within the bundle according to the policy the administrator specifies (\$4).

By not terminating the end-to-end connections at the sites, Bundler achieves a key benefit: if the bottleneck congestion is due to other traffic not from the bundle, end-to-end algorithms naturally find their fair-share. It also simplifies the implementation because Bundler does not have to proxy TCP, QUIC, and other end-to-end protocols.

As shown in Figure 1, Bundler implements its source site and destination site functions in a sendbox and receivebox, respectively. The sendbox of one site pairs with the receivebox of another site when sending traffic to it. ${ }^{2}$ These two middleboxes measure congestion signals such as the round-trip time (RTT) and the rate at which packets are received, and pass these signals to a congestion control algorithm at the sendbox (§4) to dynamically compute the bundle's sending rate. We introduce a lightweight method for the coordination between the sendbox and the receivebox that does not require any per-flow state and can be deployed in a mode that forwards packets without modification. Bundler requires no changes to the end hosts or to network routers.

Our focus thus far has been to control traffic only within a given bundle and not across different bundles. Furthermore, as we will discuss in $\S 3$, there may be instances where Bundler cannot improve performance for the bundled traffic, and falls back to the status quo; i.e., the performance achieved today when queues build in the network instead of the edge. For example, when traffic between the two sites traverses different paths with different levels of congestion, Bundler will detect this and performance will revert to the status quo.

In emulated scenarios (§7), we demonstrate that Bundler successfully enables scheduling benefits. In particular, when configured to use Stochastic Fairness Queueing (SFQ), Bundler reduces the median flow completion time (FCT) of a representative flow size distribution between $28 \%$ to $97 \%$ across a variety of scenarios. Furthermore, these performance benefits are within $15 \%$ of what would be achievable if (optimal) in-network scheduling were a possibility. In

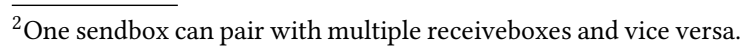

experiments over the public Internet (§8), we find that Bundler reduces short-flow latencies by $57 \%$.

\section{RELATED WORK}

Traditional congestion control. End hosts employ congestion control algorithms that aim to achieve high throughput and low delay while fairly sharing network resources with other users [24]. Each connection runs such an algorithm independently to learn about the network conditions and find the best sending rate. In Bundler, a sendbox uses such an algorithm to determine the aggregate's sending rate, rather than the rate of an individual connection. The end-hosts continue to use unmodified end-to-end congestion controllers for each connection.

Aggregating congestion information. There have been multiple proposals to aggregate congestion control information in different contexts: flows sharing the same endpoint [4], flows between two racks within a datacenter [54], and flows originating from a large cloud/content provider [43]. The goal of these approaches is to share information among the various end-to-end flows' congestion controllers, which allows them to better adapt to network conditions. Bundler has a different goal: to control queueing (and thus enable scheduling) from the edge of the network without interfering with the end-to-end congestion controllers of individual component flows. It is orthogonal to prior proposals on aggregate congestion control.

Using a middlebox for queue management. Remote Active Queue Management (AQM) [2] aims reduce VoIP traffic latency by deploying a middlebox at a site's access link that drops packets or injects ECN marks for the remaining flows in order to manipulate their end-to-end congestion control loops. It makes a core assumption that the bottleneck is the site's access link. In contrast, Bundler tackles arbitrary bottleneck locations in the middle of the network. Moreover, unlike Remote AQM, Bundler is not restricted to a specific queue management policy for a specific traffic class. 
Overlay networks. Bundler's motivation is closer to a proposal in overlay networks, OverQoS [49], which aimed to provide QoS benefits in the Internet by enforcing traffic management policies at the nodes of an already-deployed overlay network [1]. Bundler's approach is more lightweight; instead of relying on an overlay network, Bundler only requires each site to deploy a middlebox, and uses a novel control loop between the middleboxes to facilitate traffic management at the sites.

\section{GOALS AND ASSUMPTIONS}

Figure 1 describes Bundler's deployment model. Bundler aggregates traffic from Site A to Site B, and vice-versa, into two unidirectional bundles. In the egress path, the sendbox moves the in-network queues built by the bundled traffic to itself (illustrated in Figure 2) (we describe the specific mechanism in §4). It can thus enforce desired scheduling policies across the traffic in the bundle.

Our primary goal with Bundler is to provide control over self-inflicted queueing, i.e., when traffic from a single bundle causes a queue to build up at the bottleneck links in the network, even without any other cross-traffic. In the remainder of this section, we detail the conditions in which Bundler can achieve this goal. Our high-level strategy is "do no harm"; Bundler detects conditions in which it cannot operate and temporarily disables itself until favorable conditions return, reverting to status-quo performance in the meantime.

Non-edge bottleneck. Network administrators already have control over packets which queue within their site. Bundler is capable of taking control over queues that build up anywhere between a sendbox-receivebox pair. Thus, deploying Bundler at the edge of each site captures any potential build up outside of either site's control. Such congestion might occur at an inter-domain link, within either site's ISP, or, if a site is managed by a cloud provider, it could even occur within its datacenter (e.g., at the cloud provider's rate limiter, §8).

There is strong evidence that such non-edge bottlenecks exist. Dhamdhere et al. measured [15] inter-domain bottlenecks such as the red bottleneck link in Figure 1. Similarly, Zhu et al. found [53] that non-edge bottlenecks for transnational traffic to and from China are prevalent, and moreover that in many cases, the bottleneck for this traffic is an ISP deep inside China rather than a larger provider. An M-Lab technical report similarly found [50] patterns of performance degradation linked to specific ISP interconnections in the middle of the network. Finally, Jin et al. found [25] that for WAN traffic originating from Microsoft Azure, the "middle", i.e., on-path ASes not including the source or destination AS, is to blame for between $40-50 \%$ of persistent congestion incidents over a one-month period.

External congestion. Other than self-inflicted congestion, Bundler must coexist with traffic from external sources.
Congestion due to bundled cross-traffic. Bundler continues to provide benefits when the competing flows are part of other bundles from/to other sites because the rate control algorithm at each of the other sendboxes would ensure that the in-network queues remain small, and different bundles compete fairly with one another. Since each sendbox manages the self-inflicted queues for its own bundles, it can apply the appropriate scheduling policy in its per-bundle queues.

Congestion due to un-bundled cross-traffic. We now consider the scenario where the cross-traffic includes un-bundled flows. If all such un-bundled competing flows are short-lived (up to a few MBs) or application-limited (e.g., a paced video stream), the bundled traffic still sees significant performance benefits, because there are not enough packets in such short-lived flows to fill up the queues or claim a greater share of network bandwidth. However, if the cross traffic is long lasting, and employs a loss-based congestion controller to send back-logged bulk data, it aggressively fills up all available buffer space at the bottleneck link. Naively using a delay-based congestion controller at Bundler against such aggressive buffer-filling crosstraffic would severely degrade the throughput of the bundled traffic. Therefore, Bundler's congestion controller detects the presence of buffer-filling cross-traffic; to compete fairly, it relinquishes most of its control (and scheduling opportunities) over the bundled traffic, while still maintaining a small queue for continued detection of cross-traffic (detailed in §5.1). However, such pathological buffer-filling cross traffic is rare. A recent study in CDNs [5] and our analysis of a packet trace from an Internet backbone router [9] reveal that the vast majority of connections are smaller than $1 \mathrm{MB}$ : too small to build persistent queues. ${ }^{3}$ Our experiments on Internet paths (§8), also did not encounter pathological buffer-filling cross traffic.

Shared congestion across flows in the bundle. Bundler's design for moving queues via aggregate congestion control assumes that the component flows within a bundle share innetwork paths, and thus congestion. To test this assumption, we used Scamper [31] to probe all paths to 5000 random IPv4 addresses from each of 30 cloud instances across the regions of public cloud providers AWS and Azure. In no cases did we find that probe packets took different AS-level paths through the network. However, we observed instances of IP-level load balancing in $26 \%$ of IP hops. In pathological scenarios with persistent imbalance in queueing between the load-balanced paths, Bundler cannot gather accurate measurements and perform aggregated delay-based rate control for the bundled traffic. Designing a new congestion control algorithm for such scenarios remains an avenue for future work. Nonetheless, Bundler can detect these scenarios $(\S 5.2)$ and disable its rate

\footnotetext{
${ }^{3}$ This implies that flows within a bundle may also be short-lived requests or paced audio/video traffic which, when aggregated by Bundler, can form a heavy, long-lasting bundle.
} 


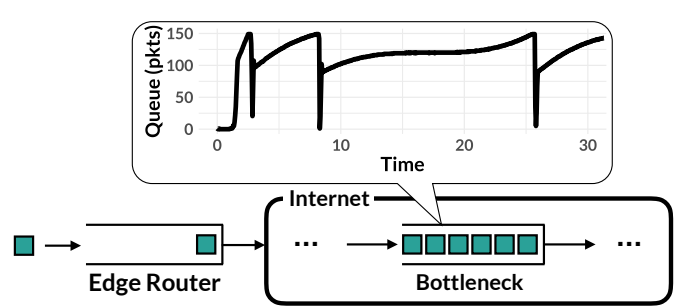

(a) Status Quo

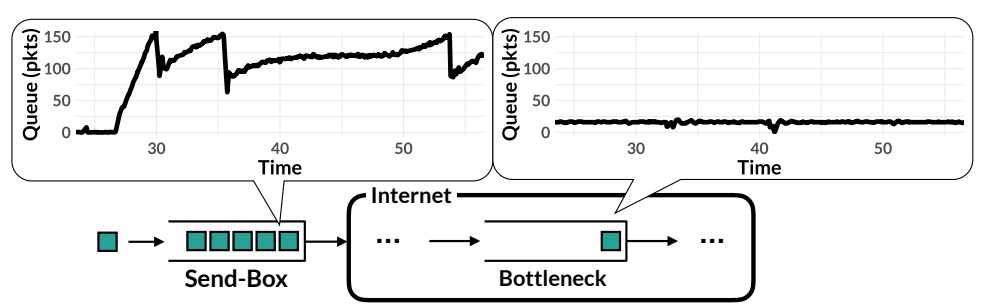

(b) With Bundler

Figure 2: This illustrative example with a single flow shows how Bundler can take control of queues in the network. The plots, from measurements on an emulated path (as in §7), show the trend in queueing delays at each queue over time. The queue where delays build up is best for scheduling decisions, since it has the most choice between packets to send next. Therefore, the sendbox shifts the queues to itself.

control in such cases, falling back to status-quo performance. We expect a well-implemented load balancer will work to prevent persistent imbalance from occurring; indeed, our success with using Bundler on real Internet paths (§8) suggests that such pathological cases do not occur in practice.

Intuition for Bundler's applicability. Another way of understanding when Bundler is useful, which incorporates the three conditions above, is the following litmus test: compare the queueing delay when all flows (Bundler's and cross-traffic) are in the network with the queueing delay when the Bundler's flows are magically removed; if the latter is lower than the former, then Bundler can provide benefits.

\section{DESIGNING BUNDLER}

Recall that in order to do scheduling, we need to move the queues from the network to the Bundler. In this section, we first describe our key insight for moving the in-network queues, and then explain our specific design choices. Recall that each site deploys one Bundler middlebox which we logically partition into sender-side (sendbox) and receiver-side (receivebox) functionality.

\subsection{Key Insight}

We induce queuing at the sendbox by rate limiting the outgoing traffic. If this rate limit is made smaller than the bundle's fair share of bandwidth at the bottleneck link in the network, it will decrease throughput. Conversely, if the rate is too high, packets will pass through the sendbox without queueing. Instead, the rate needs to be set such that the bottleneck link sees a small queue while remaining fully utilized (and the bundled traffic competes fairly in the presence of cross traffic). We make a simple, but powerful, observation: existing congestion control algorithms calculate exactly this rate [24]. Therefore, running such an algorithm to set a bundle's rate would reduce its self-inflicted queue at the bottleneck, causing packets to queue at the sendbox instead, without reducing the bundle's throughput. Note that end hosts would continue running a traditional congestion control algorithm as before (e.g., Cubic [22], BBR [10]) which

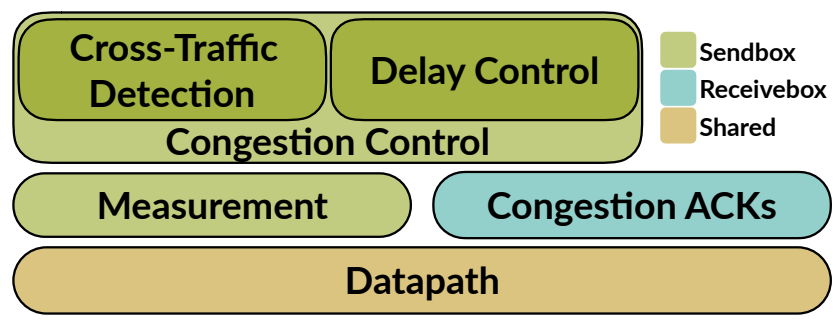

Figure 3: Bundler comprises of six sub-systems: four (in green) implement sendbox functionality, one (in blue) implements receivebox functionality, and the datapath (orange) is shared between the two.

is unaware of Bundler. Rather, the sendbox's congestion control algorithm acts on the traffic bundle as a single unit.

Figure 2 illustrates this concept for a single flow traversing a bottleneck link in the network. ${ }^{4}$ Without Bundler, packets from the end hosts are queued in the network, while the queue at the edge is unoccupied. In contrast, a Bundler deployed at the edge is able to shift the queue to its sendbox.

\subsection{System Overview}

Figure 3 shows Bundler's sub-systems: (1) A congestion control module at the sendbox which implements the rate control logic and cross-traffic detection, as discussed in §4.3. (2) A mechanism for sending congestion feedback (ACKs) in the receivebox, and (3) a measurement module in the sendbox that computes congestion signals (RTT and receive rate) from the received feedback. We discuss options for implementing congestion feedback mechanism in $\S 4.4$ and how to use that feedback in the measurement module in §4.5. (4) A datapath for packet processing (which includes rate enforcement and packet scheduling). Any modern middlebox datapath, e.g., BESS [23], P4 [6], or Linux qdiscs (as used in our prototype implementation-see §6), is suitable. We detail the interaction between these subsystems when discussing our prototype implementation in $\S 6$. In the rest of this section, we discuss our key design choices.

\footnotetext{
${ }^{4}$ Details of the emulated network setup which resulted in the illustrated
} queue length time-series are in $§ 7$. 


\subsection{Choice of congestion control algorithm}

Bundler's congestion control algorithm must satisfy the following requirements:

(1) Ability to limit network queueing. Bundler must limit queueing in the network to move the queues to the sendbox. Therefore, congestion control algorithms which are designed to control delays, and thus queueing, are the appropriate choice. A loss-based congestion control algorithm which fills buffers (e.g., Cubic, NewReno), for example, is not a good choice for Bundler, since it would build up a queue at the network bottleneck and drain queues at the sendbox.

(2) Detection of buffer-filling cross-traffic. It is well known that delay-controlling schemes (e.g., Vegas [8]) compete poorly with buffer-filling loss-based schemes [3]. Therefore, Bundler must have a mechanism to detect the presence of such competing buffer-filling flows and fall back to status quo performance, and then detect when they have left to take back its control over the network queues.

The emergence of such detection mechanisms is recent: Copa [3] detects whether it is able to empty the queues, and Nimbus [21] provides a more general mechanism which overlays a pattern on the sending rate and measures the cross traffic's response. Copa is not designed for aggregate congestion control (see §5); thus, we use the more general Nimbus mechanism.

\subsection{Congestion Feedback Mechanism}

A congestion control algorithm at the sendbox would require network feedback from the receivers to measure congestion and adjust the sending rates accordingly. We discuss multiple options for obtaining this.

Passively observe in-band TCP acknowledgements. Conventional endhost-based implementations have used TCP acknowledgements to gather congestion control measurements. A simple strategy for Bundler is to passively observe the receiver generated TCP acknowledgements at the sendbox. However, we discard this option as it is specific to TCP and thus incompatible with alternate protocols, i.e., UDP for video streaming or QUIC's encrypted transport header [29].

Terminate connections and proxy through TCP. With this approach, one would terminate end-host TCP connections at the sendbox and open new connections to the receivebox, allowing the sendbox to control the rate of traffic in these connections. This approach can improve performance by allowing end-to-end connections to ramp up their sending rates quickly. The primary disadvantage of this approach is that Bundler must take responsibility for reliable delivery of component traffic, which requires large amounts of queueing and, in the case of UDP applications, can harm application performance. Furthermore, proxying TCP connections introduces a new potential point of failure

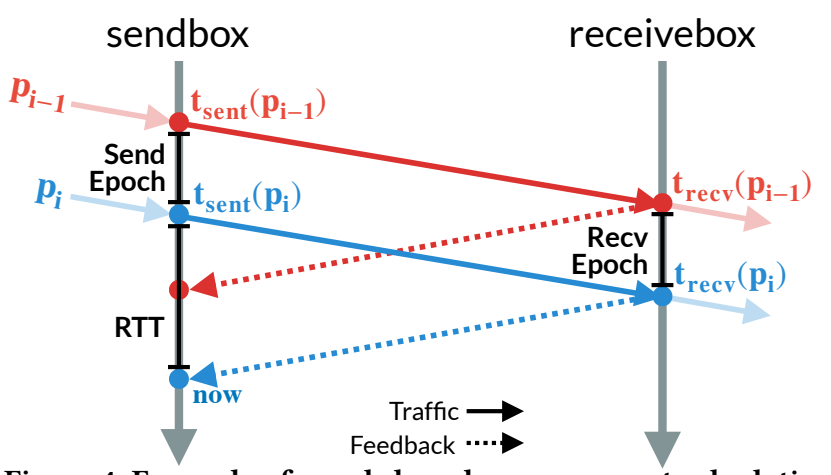

Figure 4: Example of epoch-based measurement calculation. Time moves from top to bottom. The sendbox records the packets that are identified as epoch boundaries. The receivebox, up on identifying such packets, sends a feedback message back to the sendbox, which allows it to calculate the RTT and epochs.

at Bundler that violates fate-sharing; if Bundler crashes, connections will be lost. Finally, from a practical standpoint, to avoid head-of-line blocking this approach requires that Bundler open a new proxy connection for each component end-host connection, but still determine the bottleneck rate of the traffic aggregate. While this approach may be technically feasible [4], it would result in high overhead. Thus, we set aside TCP proxies for the remainder of this discussion, but explore their compatibility with Bundler in $§ 7.5$.

Out-of-band feedback. Having eliminated the options for using in-band feedback, we adopt an out-of-band feedback mechanism: the receivebox sends out-of-band congestion ACKs to the sendbox. This decouples congestion signalling from traditional ACKs used for reliability and is thus indifferent to the underlying protocol (be it TCP, UDP, or QUIC).

\subsection{Measuring Congestion}

Sending an out-of-band feedback message for every packet arriving at the receivebox would result in high communication overhead. Furthermore, conducting measurements on every outgoing packet at the sendbox would require maintaining state for each of them, which can be expensive, especially at high bandwidth-delay products. This overhead is unnecessary; reacting once per RTT is sufficient for congestion control algorithms [36]. The sendbox therefore samples a subset of the packets for which the receivebox sends congestion ACKs. We refer to the period between two successively sampled packets as an epoch, and each sampled packet as an epoch boundary packet.

The simplest way to sample an epoch boundary packet would be for the sendbox to probabilistically modify a packet (i.e., set a flag bit in the packet header) and the receivebox to match on this flag bit. However, where in the header should this flag bit be? Evolving packet headers has proved impractical [34], so perhaps we could use 

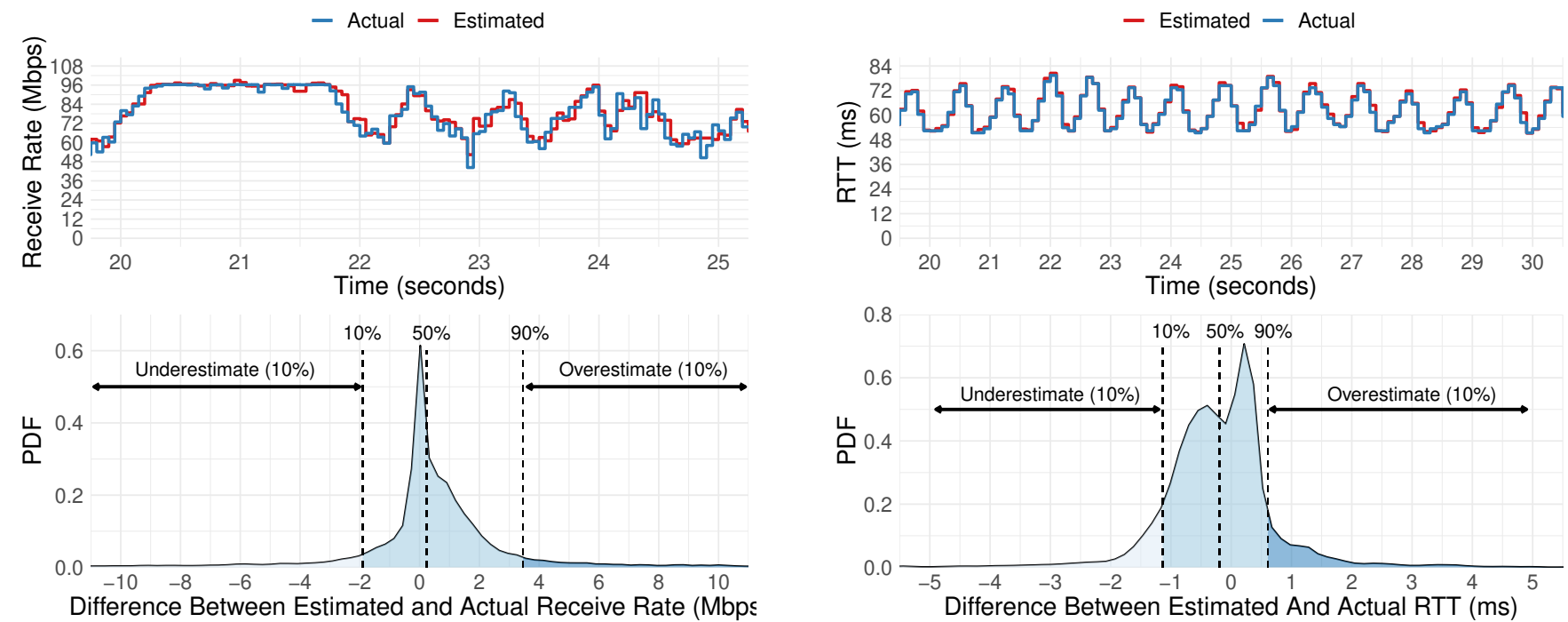

Figure 5: Bundler's estimate of the receive rate.

an encapsulation mechanism. Protocols at both L3 (e.g., NVGRE [20], IP-in-IP [41]) and L4 (e.g., VXLAN [32]) are broadly available and deployed in commodity routers today.

Happily, we observe that such packet modification is not inherently necessary; since the same packets pass through the sendbox and receivebox, uniquely identifying a given pattern of packets is sufficient to meet our requirements. In this scheme, the sendbox and receivebox both hash a subset of the header for every packet, and consider a packet as an epoch boundary if its hash is a multiple of the desired sampling period.

Upon identifying a packet $p_{i}$ as an epoch boundary packet the sendbox records: (i) its hash, $h\left(p_{i}\right)$, (ii) the time when it is sent out, $t_{\text {sent }}\left(p_{i}\right)$, and (iii) the total number of bytes sent thus far including this packet, $b_{\text {sent }}\left(p_{i}\right)$. When the receivebox sees $p_{i}$, it also identifies it as an epoch boundary and sends a congestion ACK back to the sendbox. The congestion ACK contains $h\left(p_{i}\right)$ and the running count of the total number of bytes received for that bundle. Upon receiving the congestion ACK for $p_{i}$, the sendbox records the received information, and using its previously recorded state, computes the RTT and the rates at which packets are sent and received, as in Figure 4.

Epoch boundary identification. The packet header subset that is used for identifying epoch boundaries must have the following properties: (i) It must be the same at both the sendbox and the receivebox. (ii) Its values must remain unchanged as a packet traverses the network from the sendbox to the receivebox (so, for example, the TTL field must be excluded). ${ }^{5}$ (iii) It differentiates individual packets (and not just flows), to allow sufficient entropy in the computed hash

\footnotetext{
${ }^{5}$ Certain fields, that are otherwise unchanged within the network, can be changed by NATs deployed within a site. Ensuring that the Bundler boxes sit outside the NAT would allow them to make use of those fields.
}

values. (iv) It also differentiates a retransmitted packet from the original one, to prevent spurious samples from disrupting the measurements (this precludes, for example, the use of TCP sequence number). We expect that the precise set of fields used will depend on specific deployment considerations. For example, in our prototype implementation (\$6) we use a header subset of the IPv4 IP ID field and destination IP and port. We make this choice for simplicity; it does not require tunnelling mechanisms and is thus easily deployable, and if Bundler fails, connections are unaffected. We note that previous proposals [45] have used IP ID for unique packet identification. The drawback of this approach is that it cannot be extended to IPv6. To support a wider set of scenarios, Bundler could use dedicated fields in an encapsulating header (as in [33]).

To visualize how these measurements impact the behavior of the signals over time we pick an experiment for which the median difference matches that of the entire distribution and plot a five second segment of our estimates compared to the actual values in Figure 5.

Choosing the epoch size. In order to balance reaction speed and overhead, epoch packets should be spaced such that measurements are collected approximately once per RTT [36]. Therefore, for each bundle, we track the minimum observed RTT $(\min R T T)$ at the sendbox and set the epoch size $N=(0.25 \times \min R T T \times$ send_rate $)$, where the send_rate is computed as described above. The measurements passed to the congestion control algorithms at the sendbox are then computed over a sliding window of epochs that corresponds to one RTT. Averaging over a window of multiple epochs also increases resilience to possible re-ordering of packets between the sendbox and the receivebox, which can result in them seeing different number of packets between two epochs. 
When the sendbox updates the epoch size $N$ for a bundle, it needs to send an out-of-band message to the receivebox communicating the new value. To keep our measurement technique resilient to potential delay and loss of this message, the epoch size $N$ is always rounded down to the nearest power of two. Doing this ensures that the epoch boundary packets sampled by the receivebox are either a strict superset or a strict subset of those sampled by the sendbox. The sendbox simply ignores the additional feedback messages in former case, and the recorded epoch boundaries for which no feedback has arrived in the latter.

Robust to packet loss. Note that our congestion measurement technique is robust to a boundary packet being lost between the sendbox and the receivebox. In this case, the sendbox would not get feedback for the lost boundary packet, and it would simply compute rates for the next boundary packet over a longer epoch once the next congestion ACK arrives.

Microbenchmarks. To evaluate the accuracy and robustness of this measurement technique, we picked 90 traces from our evaluation covering a range of link delays $(20 \mathrm{~ms}, 50 \mathrm{~ms}$, $100 \mathrm{~ms})$ and bottleneck rates (24Mbps, 48Mbps, 96Mbps), and computed the difference, at each time step, between Bundler's measurements (estimate) and the corresponding values measured at the bottleneck router (actual). In Figure 6 we focus on the RTT measurements: the bottom plot shows the distribution of the differences, and the top plot puts it into context by showing a five second segment from a trace where the median difference matched that of the full distribution. In Figure 5, we produce the same plots for the receive rate estimates. In summary, $80 \%$ of our RTT estimates were within $1.2 \mathrm{~ms}$ of the actual value, and $80 \%$ of our receive rate estimates were within $4 \mathrm{Mbps}$ of the actual value.

\subsection{Implications of Bundler's Design}

Our design choices result in an architecture where Bundler's inner rate control loop can be implemented entirely the "control plane" of the sendbox and the receivebox, which passively observes the packets traversing the datapath of the middleboxes, without modifying them. This results in a truly transparent system, that is light-weight, has low overhead, preserves fate-sharing, and in no way interferes with the end-to-end controllers of individual flows. The only datapath action that Bundler performs is the enforcement of the desired scheduling and queue management policies at the sendbox.

\section{UNFAVORABLE CONDITIONS}

Recall from $\S 3$ that Bundler can reliably shift queue build up from the bottleneck to itself when, (a) the cross-traffic is not buffer-filling, and (b) all of its component traffic shares the same bottleneck in the network. In practice, either of these conditions may break. In this section, we describe how
Bundler can re-use the same measurements from $\S 4.5$ to detect when these conditions do not hold. In such cases, Bundler (temporarily) disables its rate limiting (falling back to statusquo performance) until favorable conditions arise again.

\subsection{Buffer-Filling Cross Traffic}

It is well known that delay-based congestion control algorithms (as Bundler uses) lose throughput when competing with buffer-filling algorithms [3, 21]. To prevent this, Bundler utilizes prior work, Nimbus [21], which provides a mechanism for detecting the presence of buffer-filling ${ }^{6}$ cross traffic, and proposes temporarily switching to a buffer-filling scheme to compete fairly whenever such cross traffic is present. At a high-level, the detection mechanism works as follows: given a desired sending rate $r(t)$ (from an underlying congestion control algorithm), Nimbus superimposes an asymmetric sinusoid onto $r(t)$ to determine the sending rate. Then, it monitors the measured send and receive rate, estimates the cross-traffic's rate, and monitors the cross traffic's rate in the frequency domain. The sinusoidal variations in the sending rate will be visible in the cross-traffic's rate only if bufferfilling cross traffic is sharing the same bottleneck queue.

What exactly should the sendbox do when it detects buffer-filling traffic? Using a buffer-filling scheme for the bundle as in Nimbus would be fraught: since a bundle is comprised of many individual flows, the sendbox would need to know the number of flows in the bundle to know how aggressively it should compete in order to receive its fair share (as in the status quo) [13]. This number may vary significantly over time and would be difficult to measure, especially on high-performance datapaths [47].

Instead, we propose a simpler solution. Since each connection in a bundle is already employing its own congestion controller, Bundler can simply let the traffic pass, i.e., increase the pacing rate at the sendbox to stop controlling queues. Then, the end-host congestion control loops will naturally compete fairly with the buffer-filling cross traffic, just as they would without Bundler.

However, letting the traffic pass creates a new challenge. To determine when it is safe to resume delay-control while in the traffic-passing mode, Nimbus requires a superimposed pulse in both modes. If we naively let the traffic pass, the sendbox queue would never build. As a result, there would not be sufficient packets in the queue to perform the rate increase for the up-pulse. Without the up-pulse, once the sendbox switched to the buffer-filling mode, it would not be able to

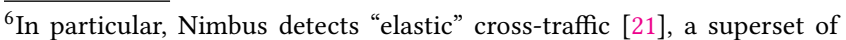
buffer-filling traffic. [21] provides an explanation of this distinction and a detailed evaluation of Nimbus' accuracy of detecting elastic cross traffic and speed of switching between the two modes, using both emulated and real-world experiments. Bundler's use of Nimbus does not impact its accuracy or speed of switching.
} 
gather sufficient information to switch back to delay-control mode once the buffer-filling cross traffic subsided.

To support the Nimbus pulses while also letting the traffic pass, the sendbox must maintain sufficient queueing for the up-pulse, i.e., the area under the up-pulse curve: $A \int_{0}^{\frac{T}{4}} \sin \left(\frac{4 \pi t}{T}\right) d t=\frac{A T}{2 \pi}$. From Nimbus, we use $T=0.2$ seconds and $A=$ one-fourth the bottleneck bandwidth $(\mu)$, which yields $\frac{T \mu}{8 \pi}$, or $8 \mathrm{~ms} \cdot \mu$ of queueing. We thus configure the sendbox to maintain a target queue $q_{T}$ of $10 \mathrm{~ms}$ (the additional $2 \mathrm{~ms}$ is a cushion against input variance). Because bundled connections will experience this queueing in addition to other queueing in the network, most traditional congestion control algorithms (e.g., Cubic) will observe RTT inflation. In $§ 7.3$ we show that this effect is not large; Bundler still achieves performance comparable to the status quo. Nevertheless, it is desirable minimize this inflation and be as close to $q_{T}$ as possible.

Thus, to achieve the target queue $q_{T}$ we use a PI controller at the sendbox which determines how the base sending rate $r(t)$ should be updated: $\dot{r}(t)=\alpha\left(q(t)-q_{T}\right)+\beta(\dot{q}(t))$, where $\dot{r}(t)$ is the update to $r(t)$ before imposing the Nimbus pulse, $q(t)$ is the current queue size at the sendbox, and $\alpha$ and $\beta$ are both positive. If $q(t)>q_{T}$, the first term will be positive and the rate will increase, causing the queue to shrink. Similarly, if the queue size is growing, the second term will be positive, which means the rate will increase and the queue will shrink. Setting $\alpha$ and $\beta$ controls a tradeoff: with larger values the controller will approach the target faster, but if they are too large the controller's variations will dominate the Nimbus pulse. If they are too small, it will take too long to reach the target. We found that $\alpha=10$ and $\beta=10$ work well for the scenarios in our evaluation ( $\$ 7$ and $\S 8$ ).

\subsection{Imbalanced Multipathing}

Since a bundle contains many component connections, a load balancer may send them along different paths. If the load along different paths is well-balanced, Bundler will accurately treat a load-balanced bottleneck link as a single link whose rate is the sum of the rates of each sub-link. However, when the load along different paths is imbalanced, the series of measurements Bundler collects will be a random sampling of the different paths, which would confuse the delay-control algorithm and cause it to perform poorly. Fortunately, such cases are straight-forward to detect with our measurement technique. More specifically, load imbalance will result in many epoch measure packets arriving out-of-order at the receivebox (whenever epoch packet $i$ happens to traverse a path with a larger delay than epoch packet $i+1)$, and consequently, out-of-order "congestion ACKs" at the sendbox. Figure 7 demonstrates this in an emulated imbalance scenario.

Therefore, we use the fraction of epoch measurement packets that arrive out-of-order as an indicator of load imbalance due to multipathing. If this number is small, the

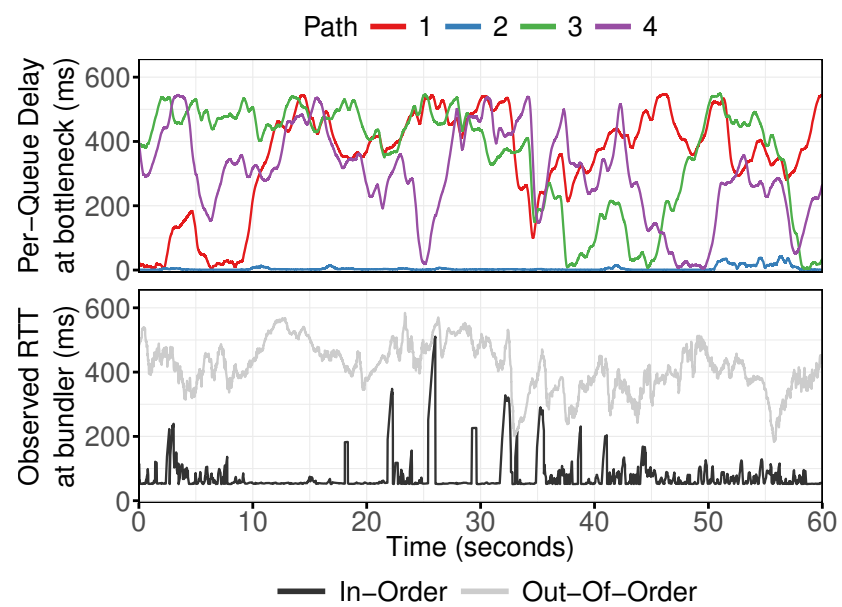

Figure 7: (Top) True delay for all packets of Bundler's component flows based on which of 4 load-balanced paths they traversed (unknown to Bundler). (Bottom) Delay measurements observed by Bundler, colored based on whether they were derived from an in-order or out-of-order epoch packet. Bundler's measurements cannot distinguish how many paths there are, but the relative number of out-of-order measurements is enough to clearly indicate the presence of multiple RTT-imbalanced paths.

links are roughly balanced and Bundler will operate as expected. If it is large, it indicates load imbalance, in which case Bundler's rate control may not work well. In $§ 7.6$, we experimentally determine an out-of-order fraction of $5 \%$ to be a good threshold indicating whether or not the links are balanced: all single-path scenarios resulted in an order of magnitude fewer out-of-order packets, and all multi-path scenarios resulted in an order of magnitude greater.

\section{IMPLEMENTATION}

Bundler boxes can be implemented as described below (although the specific implementations could vary across deployments).

Sendbox. It comprises of a data plane and a control plane. The data plane is responsible for (i) packet forwarding, (ii) tracking the number of sent bytes, (iii) identifying and reporting the epoch boundary packets to the control plane, (iv) enforcing a sending rate (computed by the control plane) on a bundle, and (iv) enforcing the desired scheduling policies for a bundle. It can implemented in software [23, 26, 28, 40], or in programmable hardware [6]. The control plane, implemented in software, is responsible for (i) measuring congestion signals using the information provided by the data plane along with the feedback from the receivebox, (ii) computing and communicating epoch sizes, and (iii) running the congestion control algorithm for each bundle to compute appropriate sending rates based on the measured congestion signals. 


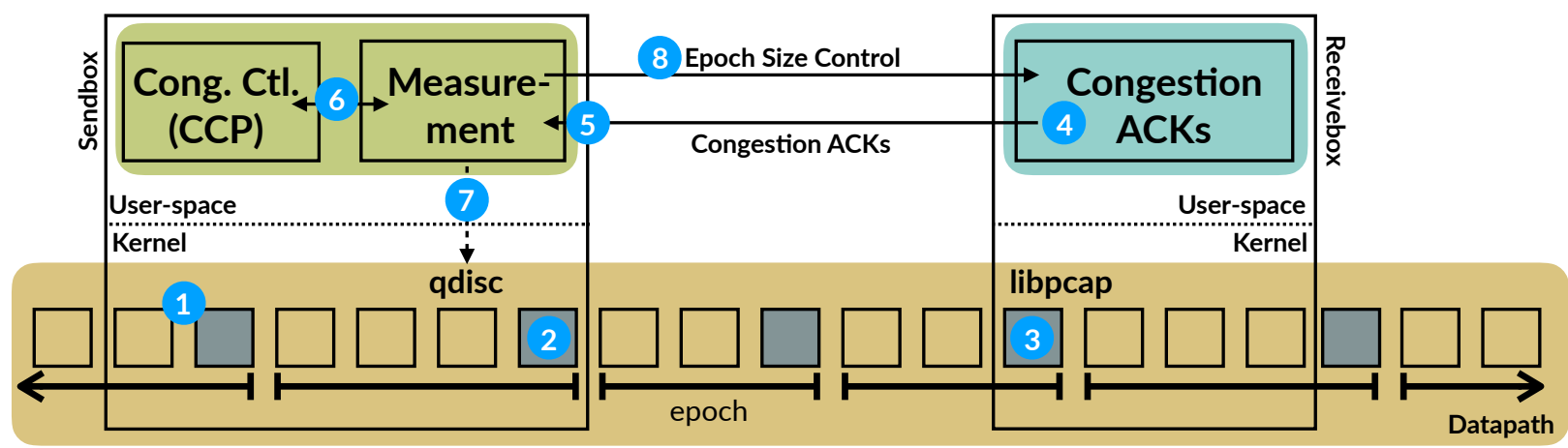

Figure 8: Bundler Implementation Overview. Box colors correspond to the roles described in Figure 3. Shaded packets are those that meet the epoch boundary condition. Dashed arrows represent communication via IPC, while solid arrows represent communication over the network.

Receivebox. It (i) tracks the number of received bytes, (ii) receives and updates epoch size values, (iii) identifies epoch boundary packets and sends feedback message to the sendbox up on receiving one. Similar to sendbox's data plane, it can also be implemented using either software or hardware.

\subsection{Prototype}

We now describe our prototype implementation of Bundler.

Sendbox data plane. We implement it using Linux tc [28] We patch the TBF queueing discipline (qdisc) [27] to detect epoch boundary packets and to report them to the control plane using a netlink socket. We use the FNV hash function [19], a non-cryptographic fast hash function with a low collision rate, to compute the packet header hash for identifying epoch boundaries. This hash function, comprising 4 integer multiplications, is the only additional per-packet work the data plane must perform to support Bundler; in our experiments, it had negligible CPU overhead.

We patch TBF's inner_qdisc to support any qdisc-based traffic controller. By default, TBF instantaneously re-fills the token bucket when the rate is updated; we disable this feature to avoid rate fluctuations caused by our frequent rate updates. Our patch to the TBF qdisc comprises 112 lines of C.

Sendbox control plane. We implement it to run in userspace in 1365 lines of Rust. We use CCP [36] to run different congestion control algorithms (described next). CCP is a platform for expressing congestion control algorithms in an asynchronous format, which makes it a natural choice for our epoch-based measurement architecture. The control plane uses libccp [36] to interface with the congestion control algorithm, and libnl to communicate with the qdisc.

Congestion control algorithms. We use existing implementations of congestion control algorithms (namely, Nimbus [21], Copa [3] and BBR [10]) on CCP to compute sending rates at the sendbox. If the algorithm uses a congestion window, the sendbox computes an effective rate of $\frac{C W N D}{R T T}$ and sets it at the qdisc. We validated that our implementation of these congestion control schemes at the sendbox closely follows their implementation at an endhost.

\subsection{Bundler Event Loop}

Figure 8 provides an overview of how our Bundler implementation operates on an already-established bundle.

(1) In the datapath, packets arrive at the sendbox qdisc. (2) The qdisc determines whether a packet matches the epoch boundary condition (§4.5). If so, it sends a netlink message to the control plane process running in user-space, and then forwards the packet normally (note the datapath does not send packets to user-space). (3) The receivebox observes the same epoch boundary packet via libpcap. (4) It sends an out-of-band UDP message to the sendbox that contains the hash of the packet and its current state. (5) The sendbox receives the UDP message, and uses it to calculate the epochs and measurements as described in §4.5. (6) Asynchronously, the sendbox control plane invokes the congestion control algorithm every 10ms [36] via libccp, (7) The sendbox control plane communicates the rate, if updated, to the qdisc using libnl. Finally (8), if the sendbox changes the desired epoch length based on new measurements, it communicates this to the receivebox, also out-of-band.

\section{EVALUATION}

Given Bundler's ability to move the in-network queues to the sendbox (as shown earlier in Figure 2), we now explore:

(1) Where do Bundler's performance benefits come from? We discuss this in the context of improving the flow completion times (FCTs) of Bundler's component flows. (\$7.2)

(2) Do Bundler's performance benefits hold across different scenarios? (\$7.3)

(3) Can Bundler work with different congestion control algorithms (\$7.4)?

(4) Are Bundler's core ideas still applicable with other design decisions? (\$7.5)

(5) Is Bundler’s heuristic ( $\$ 5.2)$ for detecting imbalanced multipath scenarios robust? (\$7.6) 
(6) Can Bundler effectively control the queues on real Internet paths? (§8)

\subsection{Experimental Setup}

We use network emulation via mahimahi [37] to evaluate our implementation of Bundler in a controlled setting; we present results on real Internet paths in $\S 8$. There are three 8-core Ubuntu 18.04 machines in our emulated setup: (1) runs a sender, (2) runs a sendbox, and (3) runs both a receivebox and a receiver. We disable both TCP segmentation offload (TSO) and generic receive offload (GRO) as they would change the packet headers in between the sendbox and receivebox, which would cause inconsistent epoch boundary identification between the two boxes. Nevertheless, throughout our experiments CPU utilization on the machines remained below $10 \%$.

Unless otherwise specified, we emulate the following scenario. A many-threaded client generates requests from a request size CDF drawn from an Internet core router [9] and assigns them to one of 200 server processes. The workload is heavy-tailed: $97.6 \%$ of requests are $10 \mathrm{~KB}$ or shorter, and the largest $0.002 \%$ of requests are between $5 \mathrm{MB}$ and $100 \mathrm{MB}$. Each server then sends the requested amount of data to the client and we measure the FCT of each such request. The link bandwidth at the mahimahi link is set to $96 \mathrm{Mbps}$, and the RTT is set to $50 \mathrm{~ms}$. The requests result in an offered load of $84 \mathrm{Mbps}$.

The endhost runs Cubic [22], and the sendbox runs Copa [3] (we test other schemes in §7.4) with Nimbus [21] for cross traffic detection. The sendbox schedules traffic using the Linux kernel implementation of Stochastic Fairness Queueing (SFQ) [35], though we briefly evaluate other policies in \$7.2. Each experiment is comprised of $1,000,000$ requests sampled from this distribution, across 10 runs each with a different random seed.

\subsection{Understanding Performance Benefits}

We first present results for a simplified scenario without any cross-traffic, i.e., all traffic traversing through the network is generated by the same customer and is, therefore, part of the same bundle. This scenario highlights the benefits of using Bundler when the congestion on the bottleneck link in the network is self-inflicted. We explore the effects of congestion due to other cross-traffic in §7.3.

Using Bundler for fair queueing. In this section, we evaluate the benefits provided by doing fair queuing at the Bundler, and use median slowdown as our metric, where the "slowdown" of a request is its completion time divided by what its completion time would have been in an unloaded network. A slowdown of 1 is optimal, and lower numbers represent better performance.

We evaluate three configurations: (i) The "Status Quo" configuration represents the status quo: the sendbox simply forwards packets as it receives them, and the mahimahi

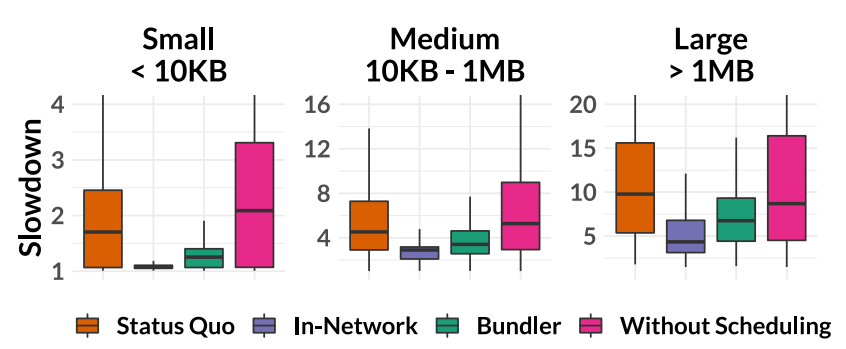

Figure 9: Bundler achieves 28\% lower median slowdown. The three graphs show FCT distributions for the indicated request sizes: smaller than $10 \mathrm{~KB}$, between $10 \mathrm{~KB}$ and $1 \mathrm{MB}$, and greater than $1 M B$. Note the different $y$-axis scales for each group of request sizes. Whiskers show $1.25 \times$ the inter-quartile range. For both Bundler and In-Network, performance benefits come from preventing short flows from queueing behind long ones. Thus, Bundler's aggregate congestion control by itself is not enough; if we configure Bundler to use FIFO scheduling, the FCTs worsen compared to the status quo.

bottleneck uses FIFO scheduling. (ii) The "In-Network" configuration deploys fair queueing at the mahimahi bottleneck. ${ }^{7}$ Recall from $\S 1$ that this configuration is not deployable. (iii) The default Bundler configuration, that uses stochastic fair queueing [35] scheduling policy at the sendbox, and (iv) Using Bundler with FIFO (without exploiting scheduling opportunity).

Figure 9 presents our results. The median slowdown (across all flow sizes) decreases from 1.76 for Baseline to 1.26 with Bundler, $28 \%$ lower. In-Network's median slowdown is a further $15 \%$ lower then Bundler: 1.07. Meanwhile, in the tail, Bundler's $99 \%$ ile slowdown is 41.38 , which is $48 \%$ lower than the Status Quo's 79.37. In-Network's 99\%ile slowdown is 27.49 .

Using Bundler for other policies. We additionally evaluated other scheduling and queue management policies with Bundler. We omit detailed results for brevity, and present a few highlights. With FQ-CoDel [16], Bundler can achieve 97\% lower median end-to-end RTTs and $89 \%$ lower 99\%ile RTTs. By strictly prioritizing one traffic class over another, Bundler results in $65 \%$ lower median FCTs for the higher-priority class.

Aggregate congestion control is not enough. It is important to note that Bundler's congestion control by itself (i.e., running FIFO scheduling) is not a means of achieving improved performance. To see why this is the case, recall that Bundler does not modify the endhosts: they continue to run the default Cubic congestion controller, which will probe for bandwidth until it observes loss. Indeed, the packets endhost Cubic sends beyond those that the link can transmit

\footnotetext{
${ }^{7}$ We implement this scheme by modifying mahimahi (our patch comprises 171 lines of $\mathrm{C}++$ ) to add a packet-level fair-queueing scheduler to the bottleneck link.
} 

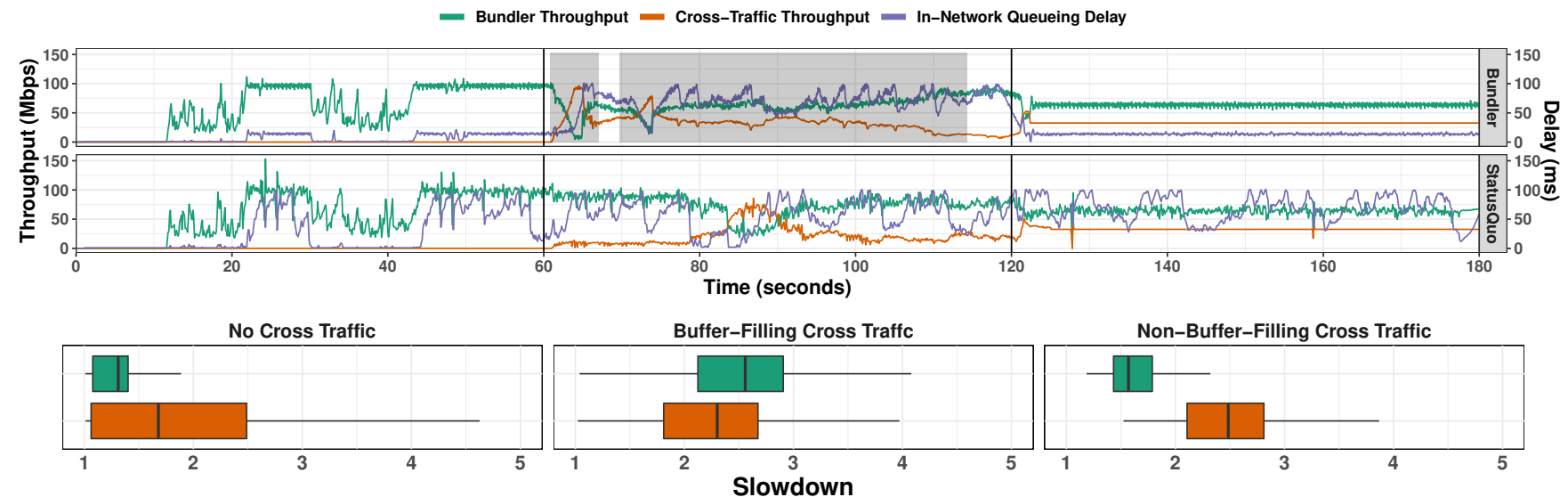

Figure 10: Bundler's scheduling ability depends on the characteristics of the cross traffic over time. In this experiment, there are 3 periods: from 0 to 60 sec., there is no competing traffic, from 60 to 120 sec. there is buffer-filling cross traffic, and from 120 to $180 \mathrm{sec}$. there is non-buffer-filling cross traffic. The box-plots below each period show the distribution of short flow FCTs during that time. During the period with buffer-filling cross traffic, Bundler detects its presence and competes fairly. The shaded region indicates time Bundler spent in buffer-filling cross-traffic mode (\$5.1).

must queue somewhere in the network or get dropped. Without Bundler, they queue at the bottleneck link; with Bundler, they instead queue at the sendbox. In addition, the delay-based congestion controller at sendbox also maintains a small standing queue at the bottleneck link (which can be seen in Figure 2) to avoid under-utilization, which increases the end-to-end-delays slightly. Therefore, doing the FIFO scheduling at the Bundler, as is done by the Status Quo, results in slightly worse performance.

\subsection{Impact of Cross Traffic}

Can Bundler successfully revert to status-quo performance in the presence of buffer-filling cross traffic, then resume providing benefits once that cross traffic leaves? In Figure 10, we show this scenario. At first, the link is occupied only by Bundler's traffic, similar to the setup described in §7.1. At time $t=60 \mathrm{sec}$, a buffer-filling cross traffic flow arrives. Bundler detects its presence (indicated by the gray shading) and starts pushing more packets into the network to compete fairly, reverting back to performance that is slightly worse than Status Quo (median FCT for short flows is $12 \%$ higher). Performance is slightly worse because of the $10 \mathrm{~ms}$ queue that Bundler continues to maintain at its sendbox for active probing to detect the cross-traffic's departure, as described in $\S 5 .{ }^{8}$ At time $t=120 \mathrm{sec}$, the buffer-filling flow stops and non-buffer-filling traffic starts, generated from the same distribution as Bundler as described in §7.1. Bundler correctly detects that it is safe to resume delay-control, and continues providing scheduling benefits. For the remainder of this subsection, we present three micro-benchmarks which dig deeper into the latter

\footnotetext{
${ }^{8}$ We believe the benefits provided by Bundler in the more common regime with no competing buffer-filling cross traffic are substantial enough to make up for slight degradation in these specific scenarios.
}

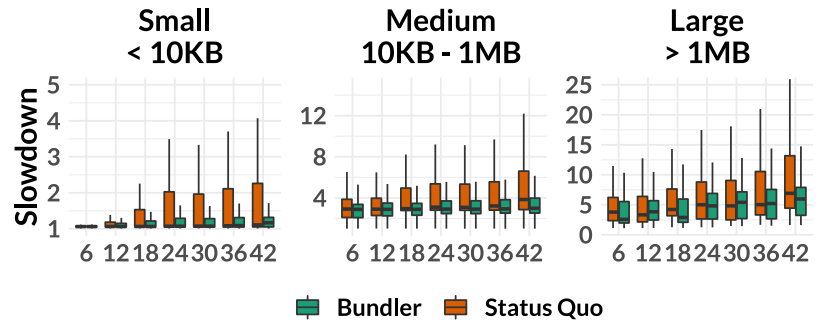

Figure 11: Against cross traffic comprising of short lived flows. Bundler offers $48 \mathrm{Mbps}$ of load to the bottleneck queue. The cross traffic's offered load increases along the $x$-axis, while Bundler's offered load remains fixed.

two scenarios, where cross traffic can affect Bundler's performance. We present results with both Nimbus and Copa being used as the congestion control algorithm at the sendbox.

Mix of flow sizes. We first consider in Figure 11 the case Bundler traffic is most likely to encounter, where the cross traffic comprises of finite-length flows up to a few MBs. We draw both Bundler's traffic and the cross traffic from the same measured distribution of web requests described in §7.1. We fix Bundler's offered load at a constant $48 \mathrm{Mbit} / \mathrm{s}$ and vary the cross traffic's offered load from 6 to $42 \mathrm{Mbit} / \mathrm{s}$.

While flows are often short, they sometimes exit slow start. With sufficient offered load, they can cause queueing in the aggregate. Observe that the Status Quo FCTs increase steadily as the cross traffic's offered load increases: this is due to the aggregate queueing effect. When this happens, Bundler's delay-based rate controller could temporarily lower its rate below the aggregate fair share of the bundled traffic. Importantly, this throughput reduction is short-lived because the queueing is short-lived, and long-term Bundler throughput is not reduced. We believe that this trade-off 


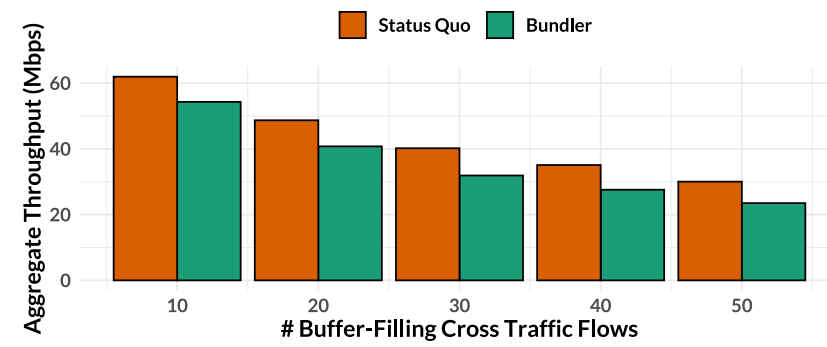

Figure 12: Varying number of competing buffer-filling cross traffic flows. Bundler controls a fixed 20 buffer-filling flows in each case.

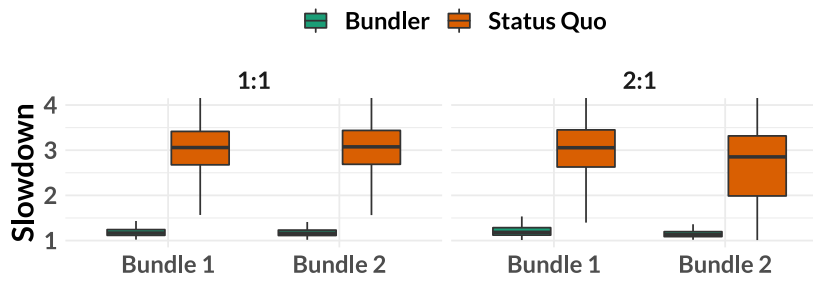

Figure 13: Competing traffic bundles. In both cases, the aggregate offered load is 84Mbps, as in Figure 9. For "1:1", we evenly split the offered load between the two Bundles; for "2:1", one bundle has twice the offered load of the other. In both cases, each bundle observes improved median FCT compared to its performance in the baseline scenario.

(short-term throughput reduction for better delay) is a good one. The lower delay helps the short flows in the bundle, while the large flows in the bundle are not affected by the short-term throughput reduction. "Mid-sized" flows in the bundle can be affected if Bundler sacrifices throughput for too long. By design, however, Bundler detects such cross-traffic and disables its delay-control mechanism in response.

Persistent elastic flows. We now evaluate how Bundler's throughput is impacted due to competition from varying amounts of persistent elastic cross-traffic. As discussed in $\S 5$, we believe this synthetic scenario is rare in practice, but when it does occur, Bundler cannot provide benefits, and since it must "hold back" some queue to detect when the cross-traffic subsides, its traffic will experience RTT inflation. Indeed, Figure 12 shows that the component flows in the bundle experience $18 \%$ less throughput on average. The impact varies from $12 \%$ lower throughput with 10 competing flows to $22 \%$ lower with 50 .

Competing Bundles. Last, we evaluate the case where flows from multiple bundles compete with one another. In Figure 13, we show the performance with two bundles of traffic competing with one another at the same bottleneck link. Both bundles comprise of web requests along with a backlogged Cubic flow. Both bundles maintain low queueing in the network and successfully control the queues at the

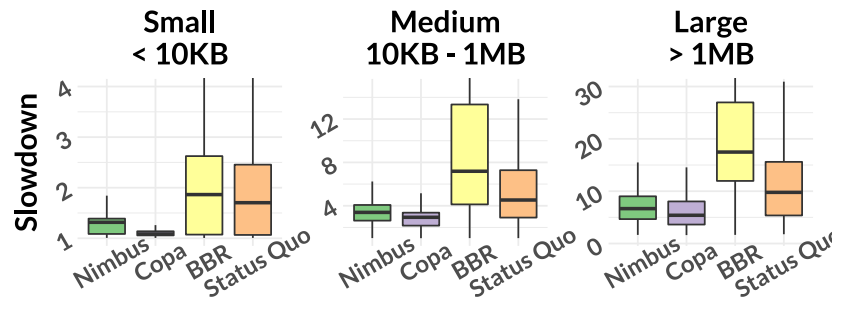

Figure 14: Choosing a congestion control algorithm at Bundler remains important, just as it is at the end-host. Note the different $y$-axis scales for each group of request sizes.

sendbox. Thus, Bundler provides benefits for both bundles, even when the amount of traffic in each bundle is different.

\subsection{Impact of Congestion Control}

We now evaluate the impact of a different congestion control algorithm running at the sendbox and at the endhosts.

Sendbox congestion control. So far we have evaluated Bundler by running Copa [3] at the sendbox. Figure 14 shows Bundler's performance with other congestion control algorithms (namely, Nimbus's BasicDelay [21] and BBR [10]), and using SFQ scheduling. We find that using BasicDelay provides similar benefits over Status Quo as Copa. BBR, on the other hand, performs slightly worse than Status Quo. This is because it pushes packets into the network more aggressively than the other schemes, resulting in a bigger in-network queue. This, combined with the queue built at the Bundler, results in the endhosts experiencing higher queueing delays than Status Quo. This shows that the choice of congestion control algorithm, and its ability to maintain small queues in the network, plays an important role.

Endhost congestion control. We used Cubic congestion control at the endhosts for our experiments so far. When we configure endhosts to use Reno or BBR, Bundler's benefits remain: Bundler achieves 58\% lower FCTs in the median compared to the updated Status Quo where the endhosts use BBR. This shows that Bundler is compatible with multiple endhost congestion control algorithms.

\subsection{Terminating TCP Connections}

Although our Bundler prototype does not terminate connections (as discussed in §4.3), we note that terminating connections does provide one key advantage: the end-to-end congestion controller will observe a smaller RTT, since the proxy can acknowledge its segments much faster than the original receiver. This enables rapid window growth at the endhosts. While there are, of course, operational concerns with managing the resulting queue, it does provide additional scheduling opportunities as well as faster ramp-up for midsized connections. 

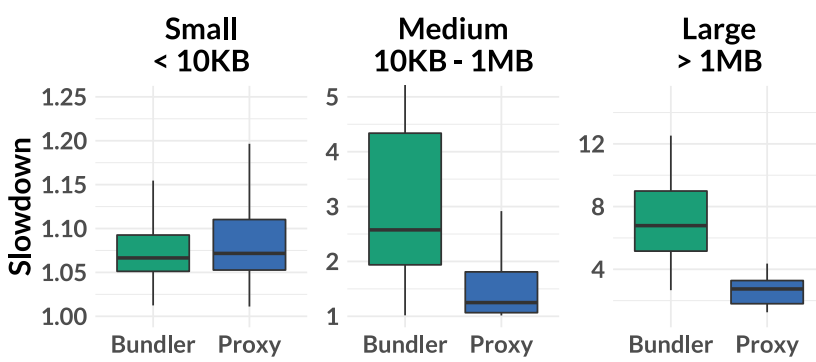

Figure 15: A proxy-based implementation of Bundler could yield further benefits to the long flows. Note the different $y$-axis scales for each group of request sizes.

How much benefit, then, could a proxy-based Bundler provide? To evaluate this, we emulate an idealized TCP proxy by modifying the endhosts to maintain a constant congestion window of 450 packets-slightly larger than the bandwidthdelay product in our setup-and increasing the buffering at the sendbox to hold these packets. The other aspects of Bundler remain unchanged. The result is in Figure 15.

For the short requests which never leave TCP slow start, terminating TCP connections does not yield additional benefits: with or without termination, they finish in a few RTTs. For medium-to-long requests, terminating TCP connections yields additional benefits since they no longer incur the penalty of window growth. Therefore, a site may benefit from proxying TCP connections at Bundler if its traffic pattern contains many medium-sized flows which benefit from fast ramp-up.

\subsection{Multipath Detection}

As described in $\S 5.2$, when the ratio of out-of-order to in-order measurements is above a certain threshold, it indicates that Bundler's component flows are likely traversing multiple imbalanced paths. To evaluate the extent to which this heuristic corresponds with imbalance, we re-run the emulation experiment from Figure 10 for a variety of network conditions (bottleneck bandwidth ranging from 12 to $96 \mathrm{Mbps}$, end-to-end RTTs ranging from 10 to 300 $\mathrm{ms}$, and bottleneck load-balancing from 1 to 32 paths) and consider the average value reported by the heuristic over each experiment. The maximum value reported across all experiments with a single path was $0.4 \%$, while the minimum value reported across all experiments with 2-32 paths was $20 \%$, two orders of magnitude greater. Thus, this heuristic provides a very clear separation between single and multiple path scenarios and a simple threshold is sufficient.

\section{REAL INTERNET PATHS}

We next evaluate our prototype implementation on real Internet paths to demonstrate that Bundler can effectively shift queues in practical settings.

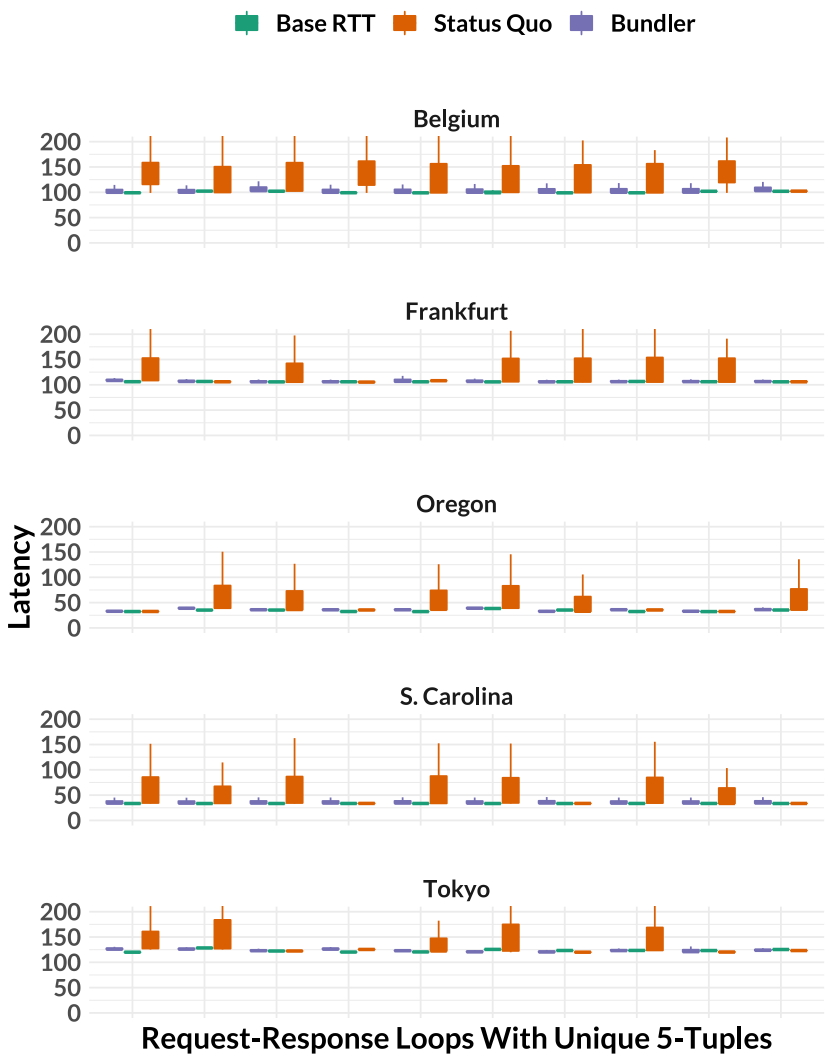

Figure 16: On 5 real-Internet paths, Bundler achieves lower latencies than Status Quo for latency-sensitive traffic. Each bar depicts an individual 5-tuple; load-balancing in the Internet prevents queueing for some 5-tuples. Bundler still offers scheduling for paths with queueing (achieving $57 \%$ lower latencies overall) while achieving overall throughput within $1 \%$ of that in the Status Quo scenario.

Experiment Setup. We deploy Bundler (sendbox) in a GCP datacenter in Iowa and generate traffic from multiple different machines in this datacenter (as detailed below). The generated traffic is sent to multiple machines in five different GCP datacenters (in Belgium, Frankfurt, Oregon, South Carolina, and Tokyo). We configured GCP to route traffic over the public Internet rather than a private network. We deploy a Bundler (receivebox) in each of these receiving datacenters, thus resulting in a total of five bundles spanning different regions of the globe.

We evaluate two different workloads in this setup: (i) Each bundle comprising of 10 parallel closed-loop 40 bytes UDP requests, where the sender issues a new request every time it receives a response. We measure the request-response RTTs in this workload to use as a baseline (and call them Base RTTs). (ii) We add 20 backlogged (iperf) flows to the above workload in each bundle. We run this workload both with and without Bundler and measure the UDP request-response RTTs (represented as Bundler and Status Quo respectively). 
Effective SFQ across all flows with Bundler should not inflate the base request-response RTT. We verified that the backlogged senders achieve similar throughput in all cases (2-4Gbit/s on these paths) both with and without Bundler, and that the Bundler machine in Iowa is not a bottleneck itself.

Result. Figure 16 shows, for each of the five bundles, the resulting RTT distributions for each of the ten request-response loops (with the 5 tuples in UDP/IP headers differing across all ten). We make two key observations: (i) The Status Quo RTTs are significantly higher than the Base RTT, which indicates significant queueing outside of either site's control. (ii) Bundler is able to move these queues and enforce SFQ scheduling effectively, resulting in request-response RTTs comparable to Base RTTs, and 57\% smaller than Status Quo at the median.

Explanation. Observation (i) above indicates that all of the conditions in $\S 3$ held during our experiment and that queues were indeed building outside of our control. One possibility is that these queues built up at an egress rate limiter imposed by GCP. However, our throughput measurements suggest that this is unlikely ${ }^{9}$; we used $\mathrm{n} 1$-standard-2 machines, which have a maximum possible egress of $10 \mathrm{Gbps}$ each, but our backlogged senders achieved only $2-4 \mathrm{Gbps}$. Nevertheless, even if queues did form at GCP's rate limiter, this represents a scenario where Bundler is useful: an operator deploying an application between multiple cloud regions could use Bundler to enforce scheduling policies on their traffic without negotiating their policy with the cloud provider or knowing where the bottleneck occurs.

\section{DISCUSSION}

Composability. Bundles are naturally composable: a sub-site within site A can deploy its own Bundler to take control of its fraction of the in-network queues, with the site A's Bundler enforcing a scheduling policy across the bundled traffic from each sub-site. For example, a department within an institute may bundle its traffic to a collaborating department in another institute, with the parent institutes bundling the aggregate traffic across multiple departments.

Scheduling across different bundles at a sendbox. We evaluate benefits of scheduling within a bundle. In practice, a given sendbox will see traffic from multiple bundles. Extending different scheduling policies to multiple such bundles can be done trivially.

Rate allocation across different competing bundles. When multiple bundles (belonging to different sites) compete at the same bottleneck, Bundler's congestion control would ensure a fair rate allocation across each of these bundles, irrespective of the amount of traffic in them. It, therefore,

${ }^{9}$ We lack visibility into Google's network and thus were unable to determine the true location of the bottleneck in this experiment. provides fairness on per-site basis, as opposed to a per-flow basis, making it more robust to popular end-host strategies such as opening multiple connections to increase bandwidth share.

\section{CONCLUSION}

We have described Bundler, a new type of middlebox which uses a novel "inner" congestion control loop for traffic bundles between two sites to shift the queues from the middle of the network, where it is difficult to unilaterally express traffic control policy, to the site itself, where doing so is tractable. Bundler neither maintains any per-flow state, nor makes any modifications to the packets. We demonstrate, using both emulated network experiments and real Internet paths, that it is possible to shift queues and schedule packets to an extent sufficient to enforce well-known scheduling disciplines.

\section{ACKNOWLEDGMENTS}

We thank Srinivas Narayana, Ahmed Saeed, Rachee Singh, the anonymous EuroSys reviewers, and our shepherd Andreas Haeberlen for their helpful discussions and feedback. This work is supported in part by DARPA contract HR001117C0048 and NSF grants 1526791, 1563826, 2006346, and 1407470. 


\section{REFERENCES}

[1] D. Andersen, H. Balakrishnan, F. Kaashoek, and R. Morris. Resilient Overlay Networks. In SOSP, 2001. 2

[2] D. Ardelean, E. Blanton, and M. Martynov. Remote Active Queue Management. In International Workshop on Network and Operating Systems Support for Digital Audio and Video, 2008. 2

[3] V. Arun and H. Balakrishnan. Copa: Congestion Control Combining Objective Optimization with Window Adjustments. In NSDI, 2018. 4.3, 5.1, 6.1, 7.1, 7.4

[4] H. Balakrishnan, H. S. Rahul, and S. Seshan. An Integrated Congestion Management Architecture for Internet Hosts. In SIGCOMM, 1999. 2, 4.4

[5] D. Berger, R. Sitaraman, and M. Harchol-Balter. AdaptSize: Orchestrating the Hot Object Memory Cache in a Content Delivery Network. In NSDI, 2017. 3

[6] P. Bosshart, D. Daly, G. Gibb, M. Izzard, N. McKeown, J. Rexford, C. Schlesinger, D. Talayco, A. Vahdat, G. Varghese, and D. Walker. P4: Programming Protocol-independent Packet Processors. SIGCOMM CCR, 44(3):87-95, July 2014. 4.2, 6

[7] R. Braden, L. Zhang, S. Berson, S. Herzog, and S. Jamin. Resource reservation protocol:(rsvp); version 1 functional specification. 1997. 1

[8] L. S. Brakmo, S. W. O’Malley, and L. L. Peterson. TCP Vegas: New Techniques for Congestion Detection and Avoidance. In SIGCOMM, 1994. 4.3

[9] CAIDA. The CAIDA Anonymized Internet Traces 2016 Dataset - 201601-21. http://www.caida.org/data/passive/passive_2016_dataset.xml, 2016. 3, 7.1

[10] N. Cardwell, Y. Cheng, C. S. Gunn, S. H. Yeganeh, and V. Jacobson. BBR: Congestion-Based Congestion Control. ACM Queue, 14(5), Oct. 2016 4.1, 6.1, 7.4

[11] D. D. Clark, S. Shenker, and L. Zhang. Supporting Real-time Applications in an Integrated Services Packet Network: Architecture and Mechanism. In SIGCOMM, 1992. 1

[12] C. Craig. ISPs do throttle traffic - and the FCC can't stop it https : / / www. infoworld. com / article / 2940538 / internet / isps - do throttle-traffic-and-the-fcc-cant-stop-it.html, 2015. 1

[13] J. Crowcroft and P. Oechslin. Differentiated End-to-end Internet Services Using a Weighted Proportional Fair Sharing TCP. SIGCOMM Comput. Commun. Rev., 28(3), July 1998. 5.1

[14] A. Demers, S. Keshav, and S. Shenker. Analysis and Simulation of a Fair Queueing Algorithm. In SIGCOMM, 1989. 1

[15] A. Dhamdhere, D. Clark, A. Gamero-Garrido, M. Luckie, R. Mok, G. Akiwate, K. Gogia, V. Bajpai, A. Snoeren, and k. claffy. Inferring Persistent Interdomain Congestion. In SIGCOMM, 2018. 1, 3

[16] E. Dumazet. CoDel - Fair Queuing (FQ) with Controlled Delay (CoDel). http://man7.org/linux/man-pages/man8/tc-fq_codel.8.html, 2012. 7.2

[17] S. Floyd. TCP and Explicit Congestion Notification. SIGCOMM CCR, 24(5), Oct. 1994. 1

[18] S. Floyd and V. Jacobson. Random Early Detection Gateways for Congestion Avoidance. IEEE/ACM Trans. on Networking, 1(4), Aug. 1993. 1

[19] G. Fowler, L. C. Noll, K.-P. Vo, D. Eastlake, and T. Hansen. The FNV Non-Cryptographic Hash Algorithm. https://tools.ietf.org/html/drafteastlake-fnv-16, 2018. 6.1

[20] P. Garg and Y.-S. Wang. NVGRE: Network Virtualization Using Generic Routing Encapsulation, 2015. RFC 7637, IETF. 4.5

[21] P. Goyal, A. Narayan, F. Cangialosi, D. Raghavan, S. Narayana M. Alizadeh, and H. Balakrishnan. Elasticity Detection: A Building Block for Delay-Sensitive Congestion Control. ArXiv e-prints, Feb. 2018. 4.3, 5.1, 6, 6.1, 7.1, 7.4

[22] S. Ha, I. Rhee, and L. Xu. CUBIC: A New TCP-Friendly High-Speed TCP Variant. ACM SIGOPS Operating System Review, 42(5):64-74, July 2008. $4.1,7.1$
[23] S. Han, K. Jang, A. Panda, S. Palkar, D. Han, and S. Ratnasamy. SoftNIC: A Software NIC to Augment Hardware. Technical Report UCB/EECS-2015-155, EECS Department, University of California, Berkeley, May 2015. 4.2, 6

[24] V. Jacobson. Congestion Avoidance and Control. In SIGCOMM, 1988. $2,4.1$

[25] Y. Jin, S. Renganathan, G. Ananthanarayanan, J. Jiang, V. N. Padmanabhan, M. Schroder, M. Calder, and A. Krishnamurthy. Zooming in on wide-area latencies to a global cloud provider. In SIGCOMM, 2019. 3

[26] E. Kohler, R. Morris, B. Chen, J. Jannotti, and M. F. Kaashoek. The Click Modular Router. ACM Trans. Comput. Syst., 18(3):263-297, Aug. 2000. 6

[27] A. N. Kuznetsov. tbf. https://linux.die.net/man/8/tc-tbf. 6.1

[28] A. N. Kuznetsov. tc. https://linux.die.net/man/8/tc. 6, 6.1

[29] A. Langley, A. Riddoch, A. Wilk, A. Vicente, C. Krasic, D. Zhang, F. Yang, F. Kouranov, I. Swett, J. Iyengar, J. Bailey, J. Dorfman, J. Roskind, J. Kulik, P. Westin, R. Tenneti, R. Shade, R. Hamilton, V. Vasiliev, W.-T. Chang, and Z. Shi. The QUIC Transport Protocol: Design and Internet-Scale Deployment. In SIGCOMM, 2017. 4.4

[30] F. Li, A. A. Niaki, D. Choffnes, P. Gill, and A. Mislove. A Large-Scale Analysis of Deployed Traffic Differentiation Practices. In SIGCOMM, 2019. 1

[31] M. Luckie. Scamper: A Scalable and Extensible Packet Prober for Active Measurement of the Internet. In IMC, 2010. 3

[32] M. Mahalingam, D. Dutt, K. Duda, P. Agarwal, L. Kreeger, T. Sridhar, M. Bursell, and C. Wright. Virtual eXtensible Local Area Network (VXLAN): A Framework for Overlaying Virtualized Layer 2 Networks over Layer 3 Networks, 2014. RFC 7648, IETF. 4.5

[33] J. McCauley, M. Zhao, E. Jackson, B. Raghavan, S. Ratnasamy, and S. Shenker. Taking an AXE to L2 Spanning Trees. In SIGCOMM, 2016. 4.5

[34] McCauley, James and Harchol, Yotam and Panda, Aurojit and Raghavan, Barath and Shenker, Scott. Enabling a Permanent Revolution in Internet Architecture. In SIGCOMM, 2019. 4.5

[35] P. E. McKenney. Stochastic Fairness Queueing. In INFOCOM, 1990. 1, 7.1, 7.2

[36] A. Narayan, F. Cangialosi, D. Raghavan, P. Goyal, S. Narayana, R. Mittal, M. Alizadeh, and H. Balakrishnan. Restructing Endpoint Congestion Control. In SIGCOMM, 2018. 4.5, 4.5, 6.1, 6.2

[37] R. Netravali, A. Sivaraman, S. Das, A. Goyal, K. Winstein, J. Mickens, and H. Balakrishnan. Mahimahi: Accurate Record-and-Replay for HTTP. In USENIX ATC, 2015. 7.1

[38] K. Nichols and V. Jacobson. Controlling Queue Delay. ACM Queue, 10(5), May 2012. 1

[39] R. Pan, P. Natarajan, C. Piglione, M. Prabhu, V. Subramanian, F. Baker, and B. VerSteeg. PIE: A Lightweight Control Scheme to Address the Bufferbloat Problem. In High Performance Switching and Routing (HPSR), 2013. 1

[40] A. Panda, S. Han, K. Jang, M. Walls, S. Ratnasamy, and S. Shenker. NetBricks: Taking the V out of NFV. In OSDI, 2016. 6

[41] C. Perkins. IP Encapsulation within IP, 1996. RFC 2003, IETF. 4.5

[42] PureVPN. ISP Bandwidth Throttling Explained. https://www.purevpn. com/blog/isp-bandwidth-throttling-explained/, 2017. 1

[43] S. Renganathan, V. N. Padmanabhan, and A. U. Nambi. Rethinking networking for five computers. In Proceedings of the 17th ACM Workshop on Hot Topics in Networks, pages 92-98. ACM, 2018. 2

[44] S. Blake and D. Black and M. Carlson and E. Davies and Z. Wang and W. Weiss. An Architecture for Differentiated Services. RFC 2475, 1998. 1

[45] Savage, Stefan and Wetherall, David and Karlin, Anna and Anderson, Tom. Practical Network Support for IP Traceback. In SIGCOMM, 2000. 4.5

[46] M. Shreedhar and G. Varghese. Efficient fair queueing using deficit round robin. In SIGCOMM, 1995. 1

[47] V. Sivaraman, S. Narayana, O. Rottenstreich, S. Muthukrishnan, and J. Rexford. Heavy-Hitter Detection Entirely in the Data Plane. In SOSR, 
2017. 5.1

[48] I. Stoica, S. Shenker, and H. Zhang. Core-stateless Fair Queueing: A Scalable Architecture to Approximate Fair Bandwidth Allocations in High-speed Networks. IEEE/ACM Trans. Netw., 2003. 1

[49] L. Subramanian, I. Stoica, H. Balakrishnan, and R. Katz. OverQoS: An Overlay Based Architecture for Enhancing Internet QoS. In NSDI, 2004. 2

[50] M.-L. R. Team et al. Isp interconnection and its impact on consumer internet performance-a measurement lab consortium technical report. https://www.measurementlab.net/publications/isp-interconnectionimpact.pdf, 2014. 3
[51] J. Wroclawski et al. The use of rsvp with ietf integrated services, 1997.1

[52] L. Zhang. Virtual Clock: A New Traffic Control Algorithm for Packet Switching Networks. In SIGCOMM, 1990. 1

[53] P. Zhu, K. Man, Z. Wang, Z. Qian, R. Ensafi, J. A. Halderman, and H. Duan. Characterizing transnational internet performance and the great bottleneck of china. Proc. ACM Measurement and Analalysis of Computer Systems, 4(1), May 2020. 3

[54] D. Zhuo, Q. Zhang, V. Liu, A. Krishnamurthy, and T. Anderson. Rack-Level Congestion Control. In HotNets, 2016. 2 